\title{
Congenital cutaneous pedicled macrocystic lymphatic malformation on the upper extremity: A rare case report and review of the literature
}

Chan Min Chung, Seung Wan Tak, Hyoseob Lim, Sang Hun Cho

Department of Plastic and Reconstructive Surgery, Hallym University Sacred Heart Hospital, Hallym University College of Medicine, Anyang, Korea
Congenital cystic lymphatic malformations on the extremities are very rare. The patient described in this case study presented with a cutaneous and pedicled macrocystic lymphatic malformation that was eliminated by electrocauterization. A 4-day-old female infant with a congenital cutaneous mass on the dorsal area of her left first metacarpophalangeal joint presented to an outpatient clinic. An electrocautery device was used to cut the pedicle gently with minimal bleeding to avoid mass rupture and to minimize morbidity. A simple wet dressing was applied for 1 week, and the wound subsequently healed completely. Cutaneous macrocystic lymphatic malformations are very rare, especially on the extremities, and no consensus exists on their treatment, which has not been previously described. This report presents this rare case, along with a review of the literature.

Keywords Cysts, lymphatic / Infant / Electrocoagulation / Lymphatic abnormalities / Skin neoplasm

\section{INTRODUCTION}

Lymphatic malformations are one of several categories of vascular tumor [1]. Tumors of lymphatic origin can exist in multiple forms. In particular, a lymphatic malformation can be microcystic, macrocystic, or mixed. Among macrocystic lymphatic malformations, cutaneous lymphatic malformations on the extremities are extremely rare. These cutaneous malformations more commonly occur in the neck, axilla, or groin area $[2,3]$.

A lymphatic malformation may cause symptoms such as infection, bleeding, and rupture, as well as cosmetic problems [4]. There-

Received: Jul 16, 2019 Revised: Aug 9, 2019 Accepted: Aug 31, 2019 Correspondence: Hyoseob Lim Department of Plastic and Reconstructive Surgery, Hallym University Sacred Heart Hospital, Hallym University College of Medicine, 22 Gwanpyeong-ro 170beon-gil, Dongan-gu, Anyang 14068, Korea

Tel: +82-31-380-3781, Fax: +82-31-380-5980, E-mail: hyoseob.lim@gmail.com Copyright @ 2019 The Korean Society for Aesthetic Plastic Surgery.

This is an Open Access article distributed under the terms of the Creative Commons Attribution Non-Commercial License (http://creativecommons.org/licenses/by-nc/4.0/) which permits unrestricted non-commercial use, distribution, and reproduction in any medium, provided the original work is properly cited. www.e-aaps.org fore, physicians who deal with lymphatic malformations should actively consider curative options. Consensus on the optimal treatment of these tumors is lacking, but many treatment methods have been attempted, such as observation, electrocauterization, aspiration, excision, and cryotherapy. Intralesional injections (sclerosing therapy), radiation, laser exposure, and ligation have also been tried [5].

Recently, we experienced a rare case of a congenital cutaneous pedicled macrocystic lymphatic malformation that was eliminated by electrocauterization.

\section{CASE REPORT}

A 4-day-old female infant with a congenital cutaneous mass on the dorsal area of her left first metacarpophalangeal joint presented to our outpatient clinic (Figs. 1, 2). Upon presentation, bleeding was noted in the area of the pedicle, but no rupture was observed. No additional lesions were identified near the mass or in surrounding areas. Clinicians considered performing additional imaging studies, including magnetic resonance imaging; however, the guardians did not provide permission for the patient to be sedated, considering that she was only 4 days old. The stalk of the mass measured 


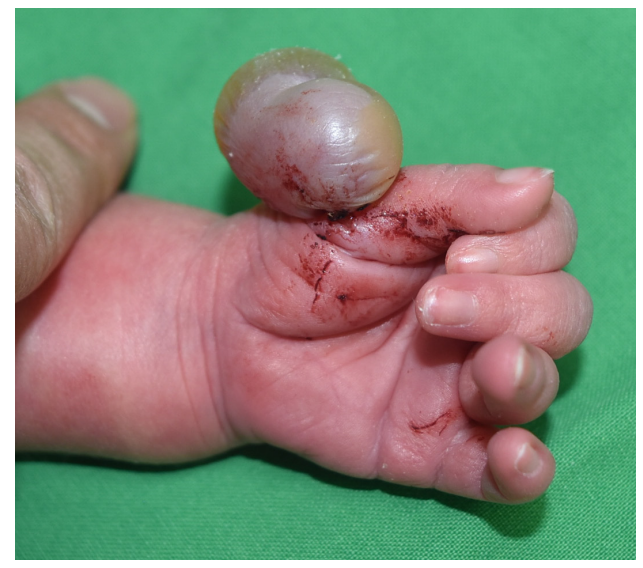

Fig. 1. Preoperative gross photograph. A macrocystic mass is observed on the patient's left hand.

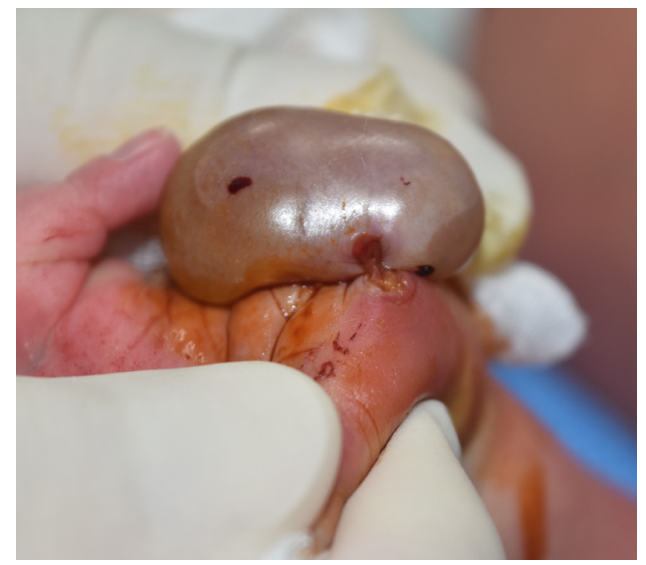

Fig. 2. Preoperative gross photograph. The mass is slightly tilted and the pedicle is shown.

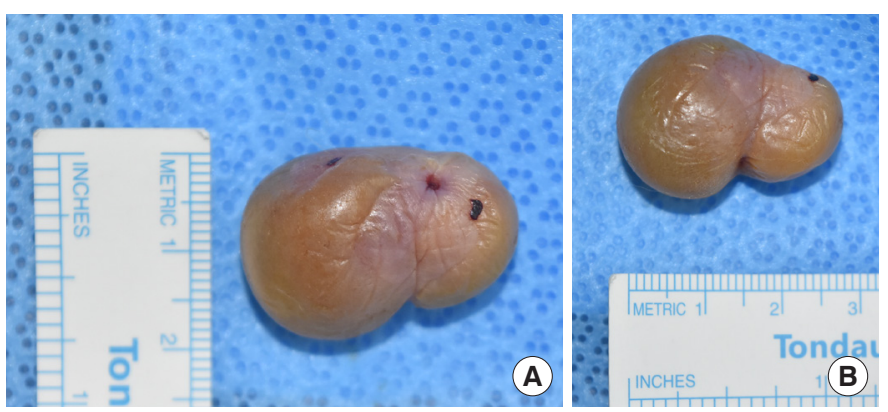

Fig. 3. Photographs of the removed lymphatic malformation. Posterior-anterior views of the removed mass are shown. (A) The height of the removed mass was measured as $1.9 \mathrm{~cm}$. (B) The width of the removed mass was measured as $2.8 \mathrm{~cm}$.

less than $2 \mathrm{~mm}$ and had no palpable inner tissue. Due to bleeding around the pedicle, the procedure was planned promptly to avoid rupture. We excised the mass without rupture, using an electrocautery device to cut the pedicle gently with minimal bleeding. The

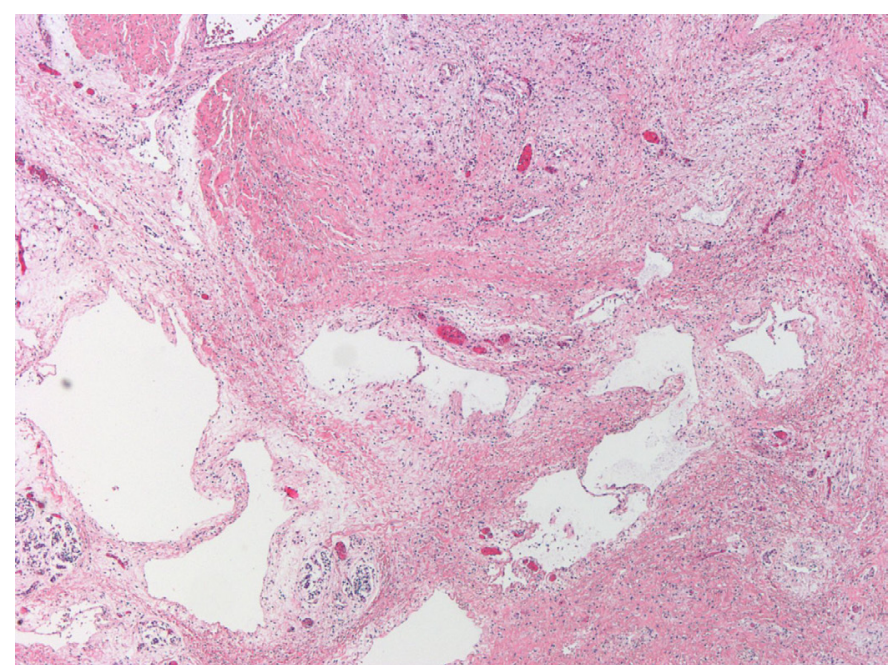

Fig. 4. Pathological findings of the lymphatic malformation. Cavernous formation of lymphatic vessels is observed (H\&E, x40).

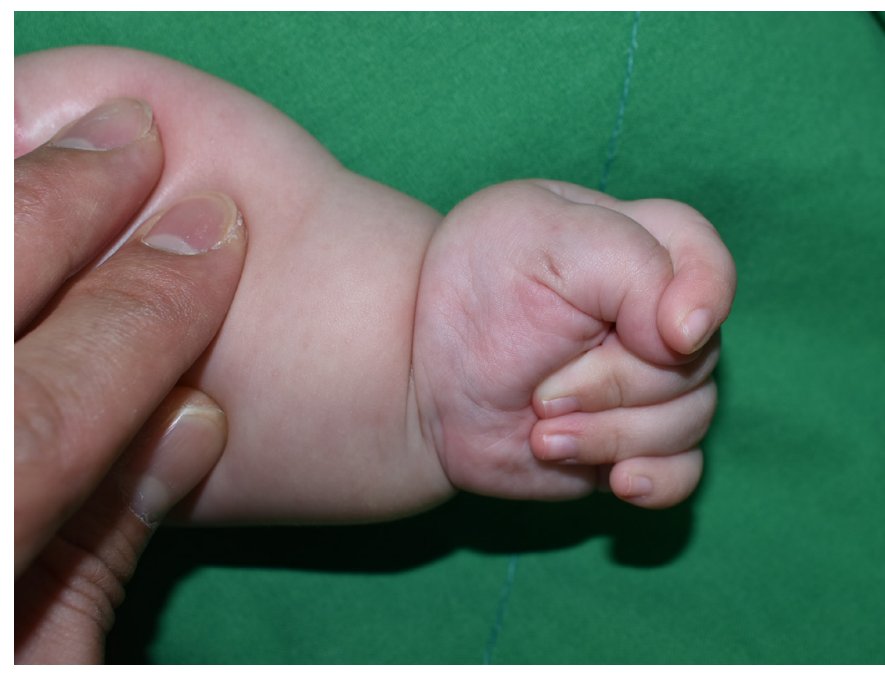

Fig. 5. Photograph taken 14 weeks post-surgery. The patient showed complete healing and no complications.

resected mass was measured as $2.8 \mathrm{~cm} \times 1.9 \mathrm{~cm}$ and was determined by a pathologist to be a lymphangioma (Figs. 3,4 ).

A simple wet dressing was applied for 1 week, and the wound subsequently healed completely. No remnants of soft tissue bulged out from the lesion where the pedicle was previously, and no humplike deformity was found. The patient presented for a follow-up appointment 3 and a half months later, and no recurrence of the deformity or lingering wound was noted at the surgical site (Fig. 5).

\section{DISCUSSION}

Tumors of lymphatic origin can be divided into microcystic, macrocystic, and mixed lymphatic malformations. Microcystic lym- 
phatic malformations are limited to the dermal region and tend to extend across a wider area. Mixed lymphatic malformations are located more deeply and are associated with diffuse swelling. These usually develop on the tongue, lips, and neck. Macrocystic lymphatic malformations also have multiform characteristics and usually appear as a bulge under the skin; these are more easily detectable than mixed lymphatic malformations [5]. Among macrocystic lymphatic malformations, cutaneous lymphatic malformations on the extremities are extremely rare. These cutaneous malformations more commonly occur in the neck, axilla, or groin area [2].

No consensus exists on the optimal treatment of cutaneous lymphatic malformations. For this reason, Ha et al. [5] concluded that treatment should be individualized according to the size and location of the tumor and the complications that might result from treatment. Flanagan and Helwig [6] came to a similar conclusion after treating 158 cases of cutaneous lymphatic malformations, in which they focused more on surgical excision. Primary surgical excision was sufficient to treat $75 \%$ of their patients. However, none of the 158 cases of cutaneous lymphatic malformations presented a pedicled cystic formation. The surgical approach in the case presented here was easier than most of the other cases reported due to its narrow stalk.

Cutaneous lymphatic malformations are easy to diagnose and cure. In contrast, other types of lymphatic malformations may be more difficult to identify and treat. For example, intra-abdominal lymphatic malformations are difficult to approach and are usually diagnosed radiologically because they are asymptomatic [7]. Furthermore, surgical treatment of patients with lymphatic malformations hidden inside the body may have higher morbidity than treatment of patients with cutaneous lymphatic malformations. For these reasons, patients with cutaneous lymphatic malformations are relatively fortunate, because aggressive treatment is possible with low morbidity.

A few reports of macrocystic lymphatic malformations exist in the literature. Flanagan and Helwig [6] reported a case of bulging on the ring and little finger of the right hand, but it was less superficial than the case described in this report. They also presented a case of a superficial lymphatic malformation that was excised not on the hand, but on the right shin [6]. Patoulias et al. [3] reported rare cystic lymphatic malformations on the inguinal and scrotal areas, but not on the extremities.

The etiology of lymphatic malformations is unclear. However, it is certain that lymphatic malformations consist of an accumulation of fluid resulting from an abnormal drainage pattern of lymphatic vessels [8]. In the head and neck region, the incidence rate of this condition is roughly 1 in 2,000 to 4,000 live births, but it is only rarely seen on the upper extremities as a macrocystic lesion [8]. Okazaki et al. [9] reported their experiences with 128 cases of lymphatic malformation, none of which were cutaneous cystic lymphatic malformations on the upper extremities. Kato et al. [10] sur- veyed 153 cases and concluded that macrocystic lymphatic malformations instead tend to occur on the head and neck area.

Diagnostic tools can be utilized to detect lymphatic malformations, such as magnetic resonance imaging, grayscale ultrasonography, or Doppler ultrasonography [8]. While no consensus currently exists on the best method of treatment of this tumor, the main option used to eliminate a malformation is surgical treatment. Ogita et al. [11] first presented intracystic injection of OK-432 (group A Streptococcus pyogenes, human origin) for sclerotherapy. Currently, molecular targeting and phototherapy are gaining increasing popularity as curative options [8]. However, for macrocystic lymphatic malformations, especially for cutaneous cases like this, surgery is still the main option. Alternatively, if the macrocystic lymphatic malformation is located more deeply, sclerosing therapy using OK-432 injection can be a good alternative.

In conclusion, this is the first report of a cutaneous macrocystic lymphatic malformation of the hand. It can manifest similarly to polydactyly, and the treatment options for these conditions are also similar. As lymphatic malformations have a high recurrence rate, surgical excision should be conducted with periodical follow-up.

\section{NOTES}

\section{Conflict of interest}

No potential conflict of interest relevant to this article was reported.

\section{Ethical approval}

The study was approved by the Institutional Review Board of Hallym Sacred Heart Hospital (IRB No. 2019-04-016-001) and performed in accordance with the principles of the Declaration of Helsinki.

\section{Patient consent}

The patient's guardian provided written informed consent for the publication and the use of the patient's images.

\section{ORCID}

Chan Min Chung

Seung Wan Tak

Hyoseob Lim

Sang Hun Cho

\section{REFERENCES}

1. Goss JA, Greene AK. Congenital vascular tumors. Otolaryngol Clin North Am 2018;51:89-97.

2. Blei F. Congenital lymphatic malformations. Ann N Y Acad Sci 2008; 1131:185-94.

3. Patoulias I, Prodromou K, Feidantsis T, et al. Cystic lymphangioma of the inguinal and scrotal regions in childhood: report of three cases. 
Hippokratia 2014;18:88-91.

4. Yang X, Jin Y, Chen H, et al. Highly selective electrocoagulation therapy: an innovative treatment for lymphangioma circumscriptum. Dermatol Surg 2014;40:899-905.

5. Ha J, Yu YC, Lannigan F. A review of the management of lymphangiomas. Curr Pediatr Rev 2014;10:238-48.

6. Flanagan BP, Helwig EB. Cutaneous lymphangioma. Arch Dermatol 1977;113:24-30.

7. Chiappinelli A, Forgues D, Galifer RB. Congenital abdominal cystic lymphangiomas: what is the correct management? J Matern Fetal Neonatal Med 2012;25:915-9.
8. Sun RW, Tuchin VV, Zharov VP, et al. Current status, pitfalls and future directions in the diagnosis and therapy of lymphatic malformation. J Biophotonics 2018;11:e201700124.

9. Okazaki T, Iwatani S, Yanai T, et al. Treatment of lymphangioma in children: our experience of 128 cases. J Pediatr Surg 2007;42:386-9.

10. Kato M, Watanabe S, Kato R, et al. Spontaneous regression of lymphangiomas in a single center over 34 years. Plast Reconstr Surg Glob Open 2017;5:e1501.

11. Ogita S, Tsuto T, Tokiwa K, et al. Intracystic injection of OK-432: a new sclerosing therapy for cystic hygroma in children. Br J Surg 1987; 74:690-1. 